\title{
Selection effects shaping the gamma ray burst redshift distributions
}

\author{
F. Fiore, D. Guetta, S. Piranomonte, V. D'Elia, and L. A. Antonelli
}

\begin{abstract}
INAF - Osservatorio Astronomico di Roma, via Frascati 33, 00040 Monteporzio Catone (Roma), Italy
\end{abstract} e-mail: fiore@oa-roma.inaf.it

Received 24 January 2007 / Accepted 13 April 2007

\section{ABSTRACT}

\begin{abstract}
Aims. Long gamma ray bursts (GRBs) are associated to the death of massive stars and have been discovered, so far, up to $z=6.29$. Therefore, they hold the promise of probing star-formation and metal enrichment up to very high redshifts. However, the present GRB samples with redshift determinations are largely incomplete, and therefore a careful analysis of selection effects plaguing these samples is mandatory before any conclusion can be drawn from the observed GRB redshift distribution.

Methods. To this purpose we study and compare three well-defined samples of long GRBs detected by Swift, HETE2, and BeppoSAX. Results. We find that Swift GRBs are, on average, slighly fainter and harder than BeppoSAX and HETE2 GRBs, as expected due to the higher energy range (15-150 keV) in which Swift GRBs are detected and localized, compared to BeppoSAX and HETE2 $(\approx 2-20 \mathrm{keV})$.

Gas and dust obscuration plays a role in shaping both the GRB samples and, most interestingly, the present samples of GRBs with redshift determination. In particular, we argue that the majority of the bright Swift GRBs without redshift might actually be $z \lesssim 2$ events and, therefore, that the present Swift GRB sample with redshift is biased against low- $z$ GRBs. On the other hand, the detection of bright UV rest-frame afterglows from high- $z$ GRBs, and even from those with large X-ray obscuration, implies a lower dust amount than in nearby GRBs and/or a different dust composition. If this is the case, the Swift sample of GRBs with redshifts is probably a fair sample of the real high- $z$ GRB population. The absence of high- $z$ GRBs in the BeppoSAX and HETE2 samples of GRBs with redshifts is probably due to how, at the time of BeppoSAX and HETE2 follow-up, faint afterglows of high-redshift GRBs will have weakened below the spectroscopic capabilities of even 10m-class telescopes.

The redshift distribution of a subsample of Swift GRBs with distributions of peak fluxes, X-ray obscuration and optical magnitude at a fixed observing time similar to those of the BeppoSAX and HETE2 samples, is roughly consistent with the real BeppoSAX+HETE2 redshift distribution.
\end{abstract}

Key words. cosmology: observations - gamma rays: observations - gamma rays: bursts

\section{Introduction}

Gamma ray bursts (GRBs) are one of the great wonders of Universe. They combine several of the hottest topics of $21 \mathrm{st}$ century astrophysics. On one hand, they are privileged laboratories for fundamental physics, including relativistic physics, acceleration processes, and radiation mechanisms. On the other, with some GRBs associated to the death of massive stars (MacFadyden \& Woosley 1999), it was soon realized, after the discovery of their cosmologic origin, that they could be used as a cosmological tool for investigating star formation and metal enrichment at the epochs of galaxy birth, formation, and growth (e.g. Wijers et al. 1998; Porciani \& Madau 2001; Fynbo et al. 2007).

In this respect, two main research areas have developed. The first one uses GRBs as background beacons for the spectroscopy of UV lines to characterize the physical and chemical state of the matter along the line of sight (Savaglio 2006, and references therein). The second includes statistical studies of the GRB redshift distributions (Guetta et al. 2005; Natarajan et al 2005; Jakobsson et al. 2006; Daigne et al. 2006). Even though the techniques adopted, therefore the reference communities, are somewhat different, these research areas are interconnected. As an example, UV lines can be used to determine the metal content of the absorption systems (D'Elia et al. 2006, 2007;
Prochaska et al. 2006; Savaglio 2006). On the other hand, it is well known that galaxy-scale properties like metallicity, starformation rate, and mass are correlated. Then, metallicity determinations obtained through GRB spectroscopy can, at least in principle, be plugged into the GRB population studies, to obtain a better constraint on the models. In this paper we concentrate on population studies and, in particular, on the importance of selection effects in shaping GRB redshift distributions.

Population studies are very powerful tools. For example, galaxy and AGN counts and luminosity functions have been used to successfully measure the evolution of the star-formation rate, as well as galaxy densities, and black-hole densities up to $z \lesssim 6$. Similarily, GRBs can be used to probe the histories of the GRB- and star- formation rates and of the metal enrichment in the Universe (e.g. Porciani \& Madau 2001). Indeed, thanks to BeppoSAX, first, and then to HETE2 and Swift we have begun to have sizable samples of GRBs with reliable redshifts (about 80 up to now). This number should grow up to 150-200 within the Swift lifetime. This opens up the possibility of computing fairly well-constrained GRB luminosity functions in a few redshift bins and, therefore, of measuring the cosmic evolution of the GRB rate. The fraction of Swift GRBs with a reliable redshift is today about one third of the total. It might be expected that this fraction will improve in future, but it will hardly approach the majority of the GRBs. This means that the biggest 

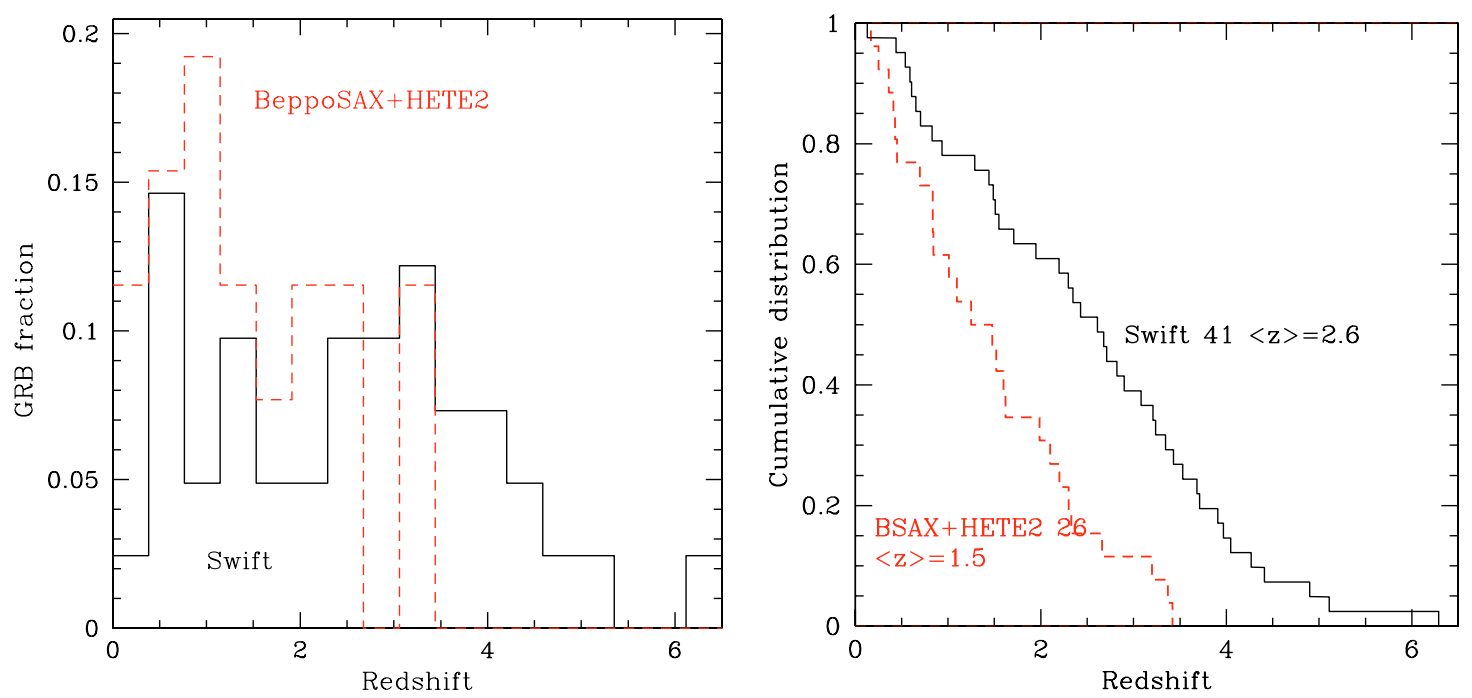

Fig. 1. Redshift distribution (left panel) and cumulative redshift distributions of Swift (solid line) and BeppoSAX+HETE2 (dashed line) GRBs.

problem we have to face in exploiting GRBs as cosmological tools is to understand and account for large selection effects. Their role in shaping the population of GRBs with a measured redshift is evident when comparing the redshift distribution of Swift GRBs with that of BeppoSAX and HETE2 GRBs (Fig. 1). The median redshifts of the two distributions are 2.6 and 1.5, respectively. This discrepancy cannot be explained simply as due to the different detector sensitivity (e.g. Guetta \& Piran 2007).

In the next sections we give a detailed description of what the possible selection effects are that plague the GRB redshift determination.

\section{Samples used in this study}

To gain more quantitavive information on the issue of GRB selection effects, we studied three well-defined samples of GRBs detected by Swift, HETE2, and BeppoSAX. We selected long GRBs $(T 90>3$ s) outside the Galactic plane to limit Galactic extinction along their line of sight and to avoid crowded fields, which can complicate the discovery of optical/NIR afterglows and thus hamper redshift determinations. To this purpose we limited our study to regions with Galactic column density along the line of sight smaller than $\times 10^{21} \mathrm{~cm}^{-2}$ (corresponding to $A_{V} \lesssim 1$ ). We also selected GRBs with good (arcmin) localization. For BeppoSAX and HETE2 GRBs, we required the $\gamma$-ray burst to be detected by the high-energy GRBM and FREGATE instruments and be localized by the WFC, WXC, or SXC instruments. For Swift we considered all long GRBs detected before September 10, 2006, while for HETE2 we considered all long GRBs detected up to December 31, 2003. For BeppoSAX we considered all GRBs detected during the entire mission. We excluded from the sample GRB 060218 and GRB980425, which are probably associated to a different class of events, orders of magnitude fainter than the rest of the sample (e.g. Guetta \& Della Valle 2007). We considered only reliable spectroscopic redshifts. Table 1 gives more information on the selected samples. Swift BAT peak fluxes and spectral parameters were taken from the Swift GRB Information page ${ }^{1}$.

Equivalent hydrogen column densities $\left(N_{\mathrm{H}}\right)$ were computed from X-ray afterglow spectra assuming solar abundances. Swift

\footnotetext{
1 http://swift.gsfc.nasa.gov/docs/swift/archive/ grb_table.html
}

column densities were taken from (Campana et al. 2006) in 17 cases, from our own analysis in 12 cases, and from the Swift GRB Information page in the rest of the cases. BeppoSAX peak fluxes and spectral parameters, including hydrogen-equivalent column densities, were taken from Stratta et al. (2004), Piro et al. (2005), and De Pasquale et al. (2006). For both samples the minimum column density is set to the Galactic value along the line of sight (Dickey \& Lockman 1990). HETE2 peak fluxes and spectral parameters were taken from Sakamoto et al. (2005).

Swift optical afterglow parameters were taken from the GCN through the Gamma Ray Burst database ${ }^{2}$. BeppoSAX and HETE2 optical afterglow parameters were taken from the Gamma Ray Burst database, from Zeh et al. (2006) and from De Pasquale et al. (2006). Table 1 gives the number of optical afterglow detections and the number of cases in which multiple observations allowed us to estimate the optical afterglow decay index. Wherever possible, we used $R$ band magnitudes. For 17 Swift GRBs and 1 BeppoSAX GRB, we have only $V$ band magnitudes, for 2 Swift GRBs only a white filter magnitude and for 8 BeppoSAX GRBs only $g$-band magnitudes. We converted the observed magnitude into the $R$ band using standard afterglow colors for all these GRBs. The Lyman- $\alpha$ forest starts to enter the $R$ band at $z=3.9$. Therefore, $R$ band magnitudes for the GRBs at $z=4-5$ should be considered lower limits. For GRB050904 at $z=6.29$, we used TAROT $I$ band equivalent magnitudes (Boer et al. 2006).

Table 1 also gives the number of reliable spectroscopic redshift obtained for the three samples. In most of the cases the redshift was obtained through absorption lines overimposed on the afterglow spectrum. In a minority of cases $(6,3$, and 2 for the Swift, BeppoSAX, and HETE2 samples, respectively), the redshift was obtained uniquely through spectroscopy of the host galaxy, where the optical afterglow was undetected or too faint to search for absorption features. In a few other cases, the redshift was obtained thanks to both absorption lines in the optical afterglow emission and host galaxy emission lines.

There are at least two large groups of selection effects that must be considered: (1) GRB detection and localization and (2) redshift determination through spectroscopy of the optical/NIR afterglow or of the GRB host galaxy. We discuss these two issues in the next sections.

\footnotetext{
${ }^{2}$ http://grad40.as.utexas.edu/grblog.php
} 
Table 1. GRB samples.

\begin{tabular}{lccccc}
\hline \hline Sat. & Tot. GRB & O.A. $^{a}$ & O. decay $^{b}$ & Tot. $z$ spec. $^{c}$ & $z$ from em. lines $^{d}$ \\
\hline Swift & 122 & 62 & 44 & 41 & 6 \\
BeppoSAX & 39 & 18 & 16 & 12 & 3 \\
HETE2 & 44 & 17 & 15 & 14 & 2 \\
\hline
\end{tabular}

${ }^{a}$ GRB with Optical Afterglows; ${ }^{b}$ GRB with multiple optical observations and estimated optical afterglow temporal decay index; ${ }^{c}$ total number of GRBs with a reliable spectroscopic redshift; ${ }^{d}$ GRBs with a redshift derived only through spectroscopy of the host galaxy.
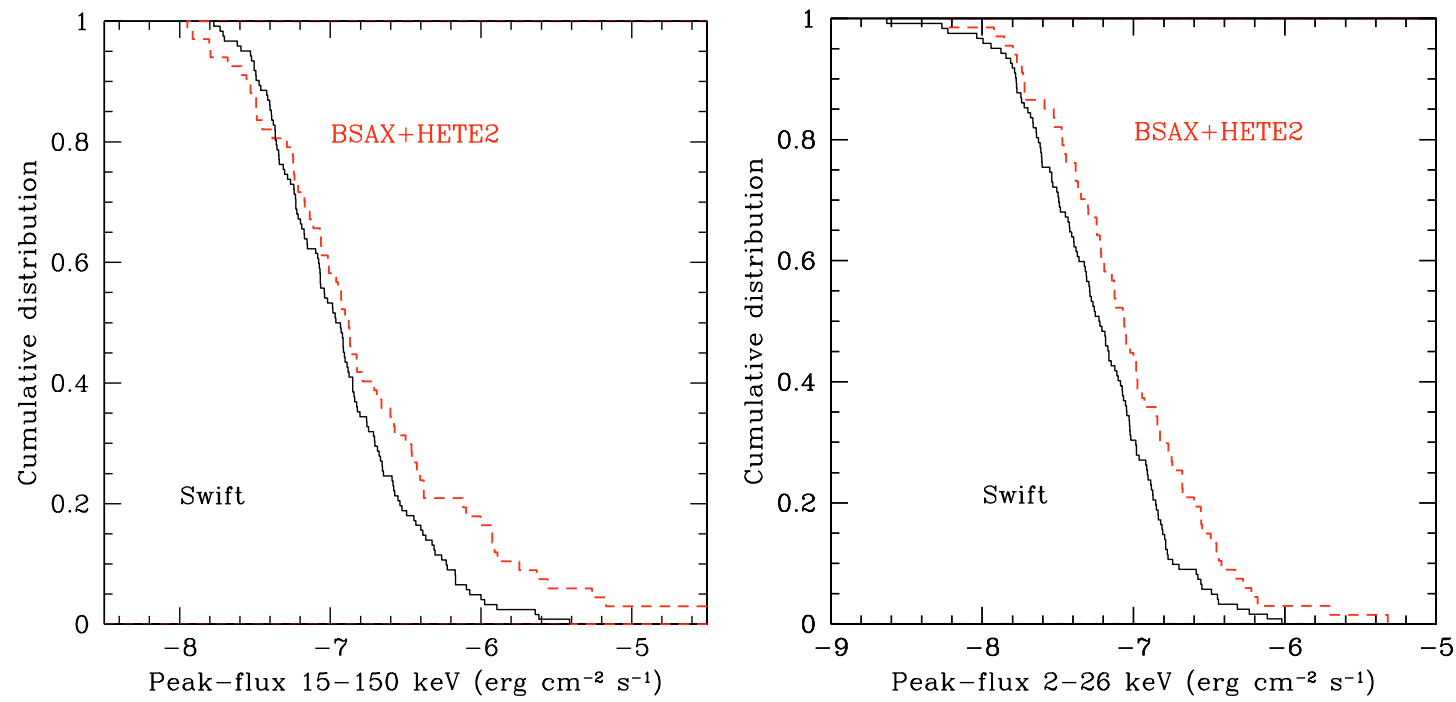

Fig. 2. peak flux cumulative distributions of the Swift (solid line) and BeppoSAX+HETE2 (dashed line) GRBs. a), left panel, 15-150 keV band; b), right panel, 2-26 keV band.

\section{GRB detection and localization}

The sensitivity of BeppoSAX, HETE2 and Swift instruments as a function of the GRB spectral shape has been studied in detail by Band (2003, 2006). Band (2006) also studied the sensitivity of the BAT instrument as a function of the combined GRB temporal and spectral properties. We refer the reader to these papers for more details on these topics.

Figure 2 compares the peak flux cumulative distributions of the Swift GRBs with that of BeppoSAX and HETE2. The comparison is made in two energy bands: $15-150 \mathrm{keV}$, which is the band where BAT detects and localizes GRBs, and 2-26 keV, which is the band where the BeppoSAX WFC and the HETE2 WXC and SXC localize GRBs. To produce Fig. 2a, BeppoSAX GRBM and HETE2 Fregate peak fluxes were converted to the $15-150 \mathrm{keV}$ BAT band by using a power-law model with an (average) energy index of 0.5 for the BeppoSAX burts and the bestfit model in Sakamoto et al. (2005) for the HETE2 bursts. To produce Fig. 2b we used WFC and WXC peak fluxes and converted BAT $15-150 \mathrm{keV}$ peak fluxes in the $2-26 \mathrm{keV}$ band by using the best-fit models and parameters and the best-fit observed column densities along the line of sight to the GRBs. To assess the robustness of our analysis we produced peak flux cumulative distributions using different, but reasonable, values of the spectral parameters adopted for the conversion from one band to the other. We always found qualitatively similar results to those in Fig. 2.

Figure 2a shows that Swift finds, on average, slightly fainter GRBs than BeppoSAX and HETE2 in the 15-150 keV band. The BeppoSAX and HETE2 samples contain a higher fraction of bright GRBs. The median $\log$ (peak flux) and its interquartile range are $-6.93,0.33$ for the Swift sample and $-6.88,0.42$ for the joined BeppoSAX+HETE2 sample. This is expected because of the higher sensitivity of the BAT instrument with respect to the BeppoSAX GRBM and HETE2 Fregate instruments (Band 2003).

The median $2-26 \mathrm{keV} \log$ (peak flux) is $-7.22,0.32$ for the Swift sample and -7.06, 0.38 for the joined BeppoSAX+HETE2 sample. The two 2-26 keV peak flux distributions differ from each other more than the $15-150 \mathrm{keV}$ distributions. This is probably due to the fact that Swift GRBs are localized at energies higher than 10-15 keV, while BeppoSAX and HETE2 GRBs are localized at energies $\lesssim 10 \mathrm{keV}$. This implies that Swift localizes, on average, harder GRBs than BeppoSAX and HETE2. In particular, Swift GRBs are revealed in a spectral range in which absorption has little, if any, effect. A column density of $N_{\mathrm{H}}=10^{23} \mathrm{~cm}^{-2}$ at $z=1$ would reduce the observed $2-10 \mathrm{keV}$ flux by $12-15 \%$ (depending on the spectral index), thus reducing the probability of detecting such highly obscured GRBs with BeppoSAX WFC and HETE2 WXC. Conversely, these GRBs would certainly be present in the Swift sample.

Figure 3a compares the best-fit column density $N_{\mathrm{H}}$ in observer frame for the samples of Swift and BeppoSAX GRBs. The X-ray afterglows at the time of the BeppoSAX NFI observations (obtained by repointing the satellite with a typical delay time of 8-10 hr from the GRB event) were significantly weaker than at the time of the Swift observations (typically minutes to a few hours after the GRB event), due to the afterglow power law decrease with exponent $\gamma=-1:-2$. This implies that the uncertainties on the X-ray spectral parameters, and therefore $N_{\mathrm{H}}$, are much greater for BeppoSAX GRBs than for Swift GRBs. Indeed, the typical uncertainty of Swift column densities is $5-10 \times 10^{20} \mathrm{~cm}^{-2}$ (see e.g. Campana et al. 2006), whereas that of BeppoSAX is $\approx 10$ times larger (see 

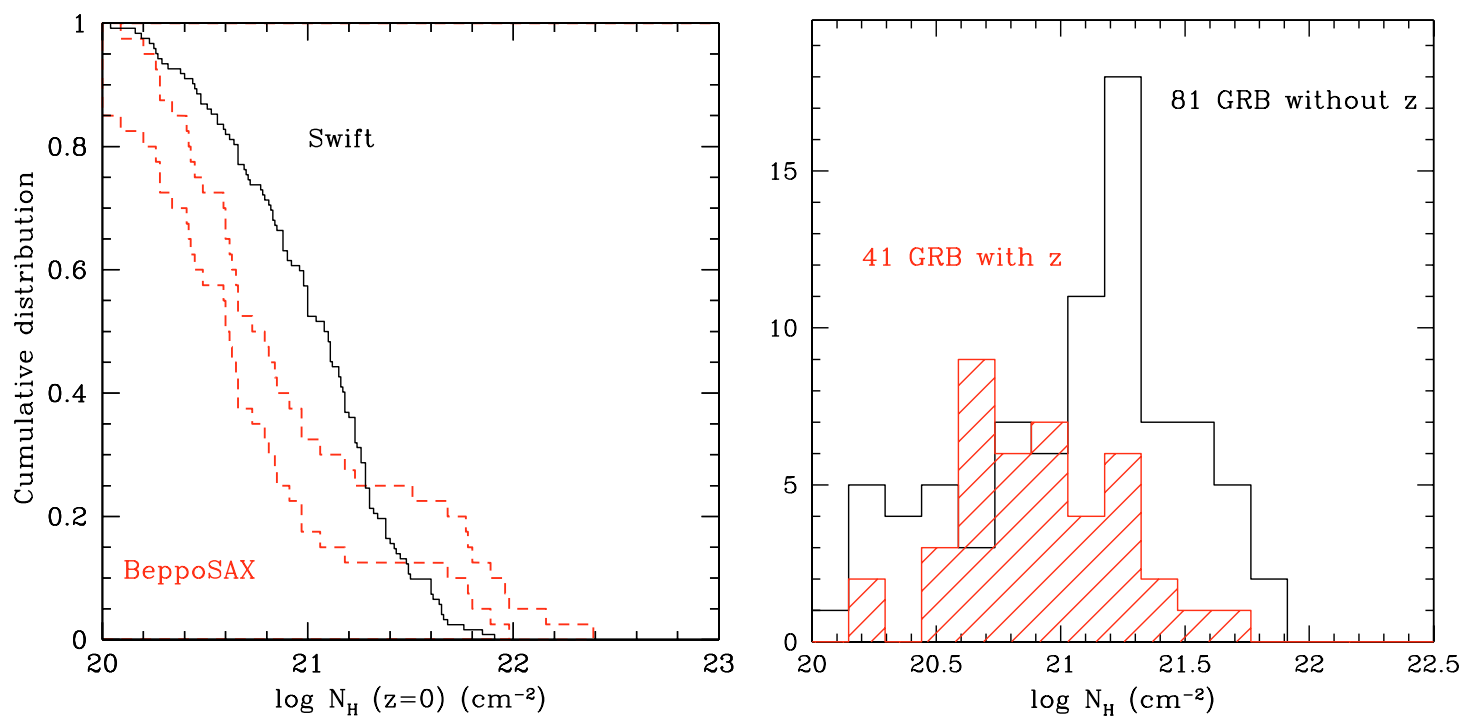

Fig. 3. a), left panel, $N_{\mathrm{H}}$ cumulative distributions of the Swift (solid line) and BeppoSAX (dashed lines) GRBs. The leftmost BeppoSAX curve assumes $N_{\mathrm{H}}=N_{\mathrm{H}}$ Galactic for the GRBs with a best-fit intrinsic $N_{\mathrm{H}}$ consistent with zero. The rightmost BeppoSAX curve assumes for these GRBs the $90 \%$ upper limit. b), right panel, $N_{\mathrm{H}}$ histograms of the Swift GRB with (shadow histogram) and without redshift (black histogram).

Stratta et al. 2004; De Pasquale et al. 2006). For this reason we plot 2 curves for the BeppoSAX GRBs. The leftmost curve assumes $N_{\mathrm{H}}=N_{\mathrm{H}}$ (Galactic) for those GRBs whose best-fit intrinsic $N_{\mathrm{H}}$ is consistent with zero. The rightmost curve is based on $90 \%$ upper limits on the $N_{\mathrm{H}}$ of these GRBs. The tail at high $N_{\mathrm{H}}$ values of this distribution is due to not very well-constrained upper limits. The real BeppoSAX $N_{\mathrm{H}}$ distribution is probabily between the two curves.

Shortward of a few $\times 10^{21} \mathrm{~cm}^{-2}$, the BeppoSAX curves in Fig. 3a are significantly lower than the Swift curve. The probability that the BeppoSAX and Swift curves are drawn from the same parent population is $<10^{-5}$ and $1.7 \%$, respectively, using the Kolmogorov-Smirnov test, thus confirming that Swift samples are less biased against obscuration than the BeppoSAX sample. Since the observer-frame column density scales as the rest-frame column density times $(1+z)$ to a high negative power ( -2.5), this implies that the BeppoSAX sample is somewhat biased against low-z, highly obscured GRBs. Conversely, these GRBs must be present in the Swift sample.

Figure $3 \mathrm{~b}$ compares the $N_{\mathrm{H}}$ distribution of the Swift GRBs with a determined redshift to that of the Swift GRBs with an undetermined redshift. The probability that the two distributions are drawn from the same parent population is only $1 \%$, suggesting that the sample of Swift GRBs with determined redshift is biased against GRB with high (observer-frame) obscuration. Indeed, the $N_{\mathrm{H}}$ distributions of the Swift and BeppoSAX GRBs with redshifts are similar, unlike the $N_{\mathrm{H}}$ distributions of the full Swift and BeppoSAX GRBs (see above). This introduces the next important group of selection effects, those related to the determination of the redshift of a GRB through spectroscopy of the optical/NIR afterglow or of its host galaxy.

\section{Redshift determination}

In determining the redshift of a GRB, the identification of the optical afterglow plays a major role. Only 6 Swift redshifts have been found through spectroscopy of the host galaxy ( 5 for the BeppoSAX and HETE2 joined sample).

Optical afterglows have been discovered for only $50 \%$ of the Swift GRB sample, a fraction only slightly greater than

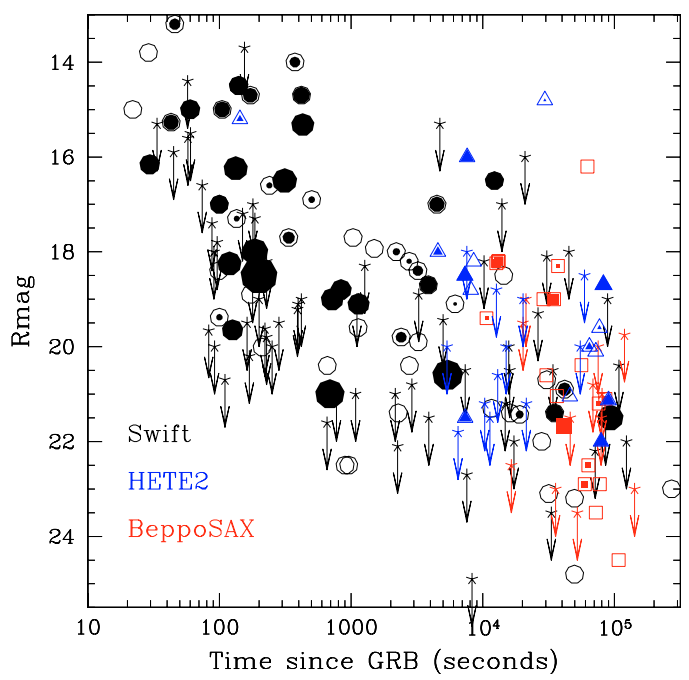

Fig. 4. The $R$ magnitude of the optical afterglow at the time of its discovery as a function of this time. Filled symbols are GRBs with reliable redshift determination. The size of the symbol is proportional to the redshift (the larger the symbol, the higher the redshift). Circles = Swift GRBs; squares = BeppoSAX GRBs; triangles = HETE2 GRBs.

that of the BeppoSAX and HETE2 samples (46\% and 39\%, respectively). This result is somewhat surprising, considering the prompt Swift localization (minutes) and the strong international effort on Swift GRB follow-up observations, which exploits an impressive number of facilities from dedicated robotic telescopes to $8 \mathrm{~m}$ class telescopes like the VLT, Gemini, and Keck. It was expected that such an effort would have produced a much larger fraction of optical/NIR afterglow identifications than BeppoSAX and HETE2.

Figure 4 shows the $R$ magnitude of the optical afterglow as a function of the discovery-time of the optical afterglow for the Swift, BeppoSAX, and HETE2 GRBs. As expected, redshifts tend to be found for bright afterglows. The figure also suggests that, at a given time from the GRB event, the Swift optical afterglows are fainter, on average, than the BeppoSAX and HETE2 afterglows. We then computed the magnitude of the 

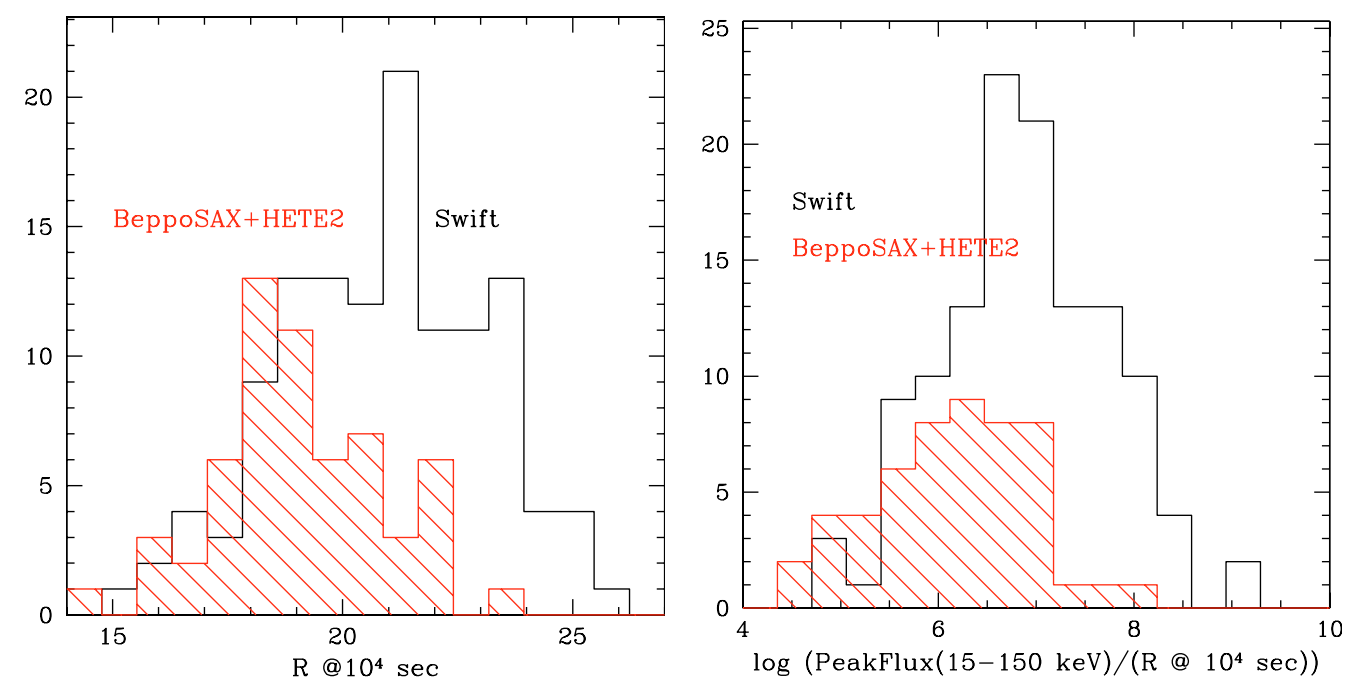

Fig. 5. a), left panel, the distribution of the $R$ magnitude $10 \mathrm{ks}$ after the GRB events for the Swift (solid histogram) and the BeppoSAX+HETE2 (dashed histogram) GRB samples. b), right panel, the distribution of the $\gamma$-ray (15-150 keV) to optical ( $R$ band) flux ratio for the Swift (solid histogram), and BeppoSAX+HETE2 (red histogram) GRB samples.

Swift, BeppoSAX, and HETE2 afterglows at a fixed time using the best-fit decay indices found for each GRB afterglow, when available. In the rest of the cases we used a time decay index of -1 . We chose a fixed time of $10 \mathrm{ks}$ after the burst (observer frame), which is intermediate between the typical times at which Swift, BeppoSAX and HETE2 GRBs are discovered, thus minimizing the extrapolation needed to compute the $R$ mag at $10 \mathrm{ks}$.

Figure 5a compares the Swift distribution of the $R$ mag at $10 \mathrm{ks}$ from the GRB event with that of the BeppoSAX and HETE2. GRBs without detection of optical afterglow but for which optical follow-up observations were carried out are included in this distribution at the magnitude of their upper limits. This figure confirms that Swift finds bursts with a fainter optical afterglow. The probability that the Swift and BeppoSAX+HETE2 distributions are drawn from the same parent population is $<10^{-5}$. Similar results are obtained by considering the distributions of the magnitudes of the detected afterglows, excluding the upper limits. In principle, the fainter Swift optical afterglows may be due to the fact that Swift detects, on average, fainter GRBs (see Fig. 2). However, this is probably not the case. Figure 5b shows the Swift and BeppoSAX+HETE2 distributions of the $\gamma$-ray $(15-150 \mathrm{keV})$ to optical ( $R$ band) flux ratio. (Also in this case, GRBs with undetected optical afterglow are included at the magnitude of their upper limits.) The probability that the two distributions are drawn from the same parent population is lower than $1 \%$. This probability increases to $1.6 \%$ by comparing the distributions of the magnitudes of the detected afterglows, excluding the upper limits. Similar results are obtained considering the X-ray $(2-26 \mathrm{keV})$ to optical flux ratio. Computing the $R$ magnitude at $1 \mathrm{ks}$ or at $100 \mathrm{ks}$ does not change this result qualitatively.

\section{Selection effects at work}

There two major differences in the Swift and BeppoSAX+HETE2 redshift distributions: a) a relatively large number of GRB with $z>3.5$ is present in the Swift sample (11 out 41 GRBs, i.e. $27 \%$ of the sample). These GRBs are absent in the combined BeppoSAX+HETE2 sample; b) a deficit of low redshift $(z \lesssim 2)$ in the Swift sample with respect to what would be expected based on the BeppoSAX+HETE2 sample. We discuss these two points in turn.

About the first point, the Swift better sensitivity to faint GRBs and the Swift quick localization may explain the presence of a large number of high-redshift GRBs in the Swift sample compared to the BeppoSAX and HETE2 samples. First, the highest redshift GRBs are found at low peak fluxes in Fig. 6a), which plots the redshift as a function of the $15-150 \mathrm{keV}$ peak flux for the Swift, BeppoSAX, and HETE2 GRB samples. Second, the Swift capability to localize the GRB on timescales of minutes allows the discovery of faint optical afterglows, which can be promptly observed in spectroscopic mode. The median delay time of optical follow-up for the Swift, HETE2, and BeppoSAX GRBs is $15 \mathrm{~min}, 3.5 \mathrm{~h}$, and $14 \mathrm{~h}$, respectively. If optical and near-infrared afterglows decrease like power laws with exponent $\gamma \approx-1$, they would have faded by 2.9 and $4.4 \mathrm{mag}$ passing from the median Swift delay time to the median HETE2 and BeppoSAX delay times, respectively. Faint afterglows of high-redshift GRBs will have weakened even below the spectroscopic capability of $10 \mathrm{~m}$ class telescopes, if observed many hours later like in the BeppoSAX and HETE2 era. Furthermore, the host galaxies of high-redshift GRBs are too faint to allow redshift determinations through their emission lines.

The discrepancy between the Swift and BeppoSAX+HETE2 samples at low redshift is less straightforward and requires a more detailed discussion. Figure 6a shows that the peak flux distrubution becomes wider at low redshift. Indeed, the median redshift of the 24 Swift GRB with $15-150 \mathrm{keV}$ peak flux $>3 \times 10^{-7} \mathrm{erg} \mathrm{cm}^{-2} \mathrm{~s}^{-1}(\sim 20 \%$ of the sample) is only $\langle z\rangle=1.5$, very different from the median redshift of the full sample $(\langle z\rangle=$ 2.6). The sample of bright GRBs is particularly useful because: a) selection effects due to temporal and spatial variations in the instrument sensitivity are minimized; and b) the redshift range is narrower, with high- $z$ GRBs systematically fainter than bright GRBs, thus minimizing evolutionary effects. For bright fluxes the sensitivity of the instruments can be safely considered constant over their entire field of view, so it is easier to compare the number of GRBs expected by different experiments. Comparing the field of view of Swift BAT to that of the BeppoSAX WFC and considering the net observing time spent by the two satellites searching for GRBs, we expect a number of bright GRBs 

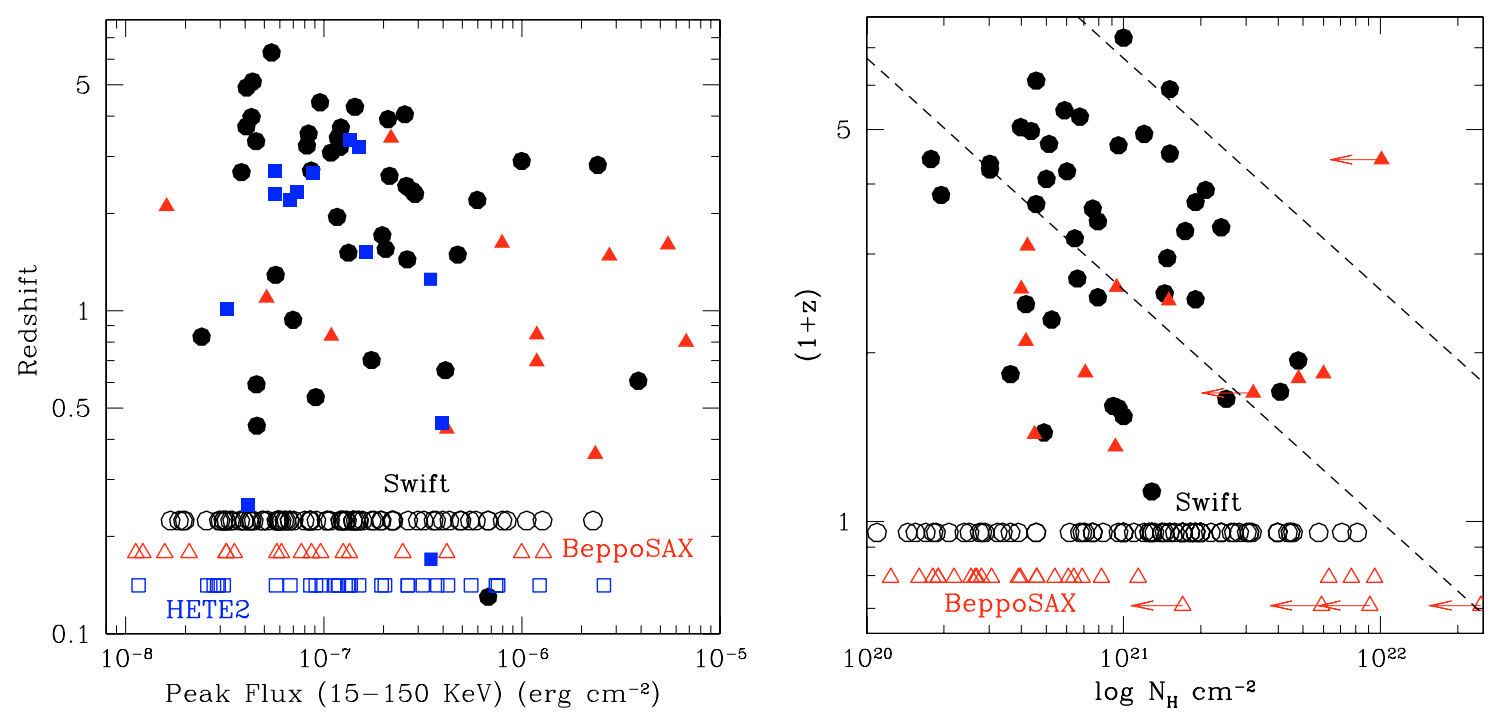

Fig. 6. The redshift as a function of the $15-150 \mathrm{keV}$ peak flux a), left panel, and of the observer frame $N_{\mathrm{H}} \mathbf{b}$ ), right panel, for the Swift (circles), BeppoSAX (triangles) and HETE2 (squares) GRBs. GRB without a measured redshift are plotted at a constant $z$ as empty symbols. The left dashed line in Fig. $6 \mathrm{~b}$ is the expectation for a constant, rest-frame column density of $\log N_{\mathrm{H}}=22$, and the right dashed line is the expectation for $\log N_{\mathrm{H}}=23$.

$\left(15-150 \mathrm{keV}\right.$ peak flux $\left.>3 \times 10^{-7} \mathrm{erg} \mathrm{cm}^{-2} \mathrm{~s}^{-1}\right)$ to be $\sim 1.5$ times higher in the Swift sample than in the BeppoSAX sample, a factor similar to what is found in the real GRB samples (1.77). Conversely, the number of bright GRBs with $z<2$ in the Swift sample is only half that in the BeppoSAX sample (4 against 8). It is clear that a strong selection effect is at work, biasing the sample of Swift GRBs with redshift against low-z sources. Indeed, only 7 out of 24 bright Swift GRBs have a spectroscopic redshift, to be compared to 8 out of 13 in the BeppoSAX sample (and 3 out of 6 in the HETE2 sample).

A possible cause of the difficulty in obtaining a redshift for many bright Swift GRBs is obscuration. The median observerframe column density toward the bright Swift GRBs is $\log N_{\mathrm{H}}=$ 21.28 with an interquartile range of 0.24 , while the median $\log N_{\mathrm{H}}$ of the faint Swift GRBs is $\log N_{\mathrm{H}}=21.0$ with interquartile 0.30 . The probability that the two $\log N_{\mathrm{H}}$ distribution are drawn from the same parent population is $\$ 2 \%$. The median $\log N_{\mathrm{H}}$ of the 13 BeppoSAX GRBs with $15-150 \mathrm{keV}$ peak flux $>3 \times 10^{-7} \mathrm{erg} \mathrm{cm}^{-2} \mathrm{~s}^{-1}$ is 20.66 (or 20.97 assuming the $90 \%$ upper limit for the GRBs with a best-fit intrinsic $N_{\mathrm{H}}$ consistent with zero). The intrinsic observer-frame $\log N_{\mathrm{H}}$ (i.e. after subtraction of the Galactic column density along the line of sight) of the 24 Swift bright GRBs is 21.10. At a typical redshift of 1.5 this implies a rest-frame column density of $\log N_{\mathrm{H}} \sim 22.1$ and an optical extinction of several magnitudes, assuming a Galactic dust-to-gas ratio. This dust extinction would make more difficult both to discovery optical afterglows and to determine the redshift through optical spectroscopy. Indeed, the fraction of detected optical afterglows among the bright Swift GRBs is $46 \%$, slightly smaller than for the sample of the 98 Swift GRBs with $15-150 \mathrm{keV}$ peak flux $<3 \times 10^{-7} \mathrm{erg} \mathrm{cm}^{-2} \mathrm{~s}^{-1}(52 \%)$. The median $R$ band magnitudes of the bright GRBs $(\langle R\rangle=18.4)$ is also similar to the faint GRBs $(\langle R\rangle=18.7)$. Nearly identical are the median $R$ magnitudes at $10 \mathrm{ks},\langle R\rangle=20.73$ for bright GRBs and $\langle R\rangle=20.72$ for the faint GRBs. Conversely, one would expect fainter optical afterglows for the fainter GRBs. Finally, the fraction of bright Swift GRB with redshift is only $29 \%$, while that of bright BeppoSAX and HETE2 GRBs is $62 \%$ and $50 \%$, respectively, despite the much quicker optical followup observations for Swift GRBs. Excluding the objects with redshift obtained from host-galaxy emission lines from these samples does not change this conclusion.

Figure $6 \mathrm{~b}$ plots the redshift as a function of the observer frame $N_{\mathrm{H}}$ for the Swift and BeppoSAX GRB samples. Not surprisingly, the highest redshift GRBs are found not only at low peak fluxes (Fig. 6a), but also at low observed column densities. The two dashed lines in Fig. 6b) are the expectation of a constant, rest-frame column density of $\log N_{\mathrm{H}}=22$ (left line) and $\log N_{\mathrm{H}}=23$ (right line). The observed Swift $\log N_{\mathrm{H}}$ distribution is consistent with the expectation of rest-frame column densities of the order of $10^{22} \mathrm{~cm}^{-2}$, typical of dense molecular clouds. GRBs with rest-frame obscuring column densities of the order of $10^{23} \mathrm{~cm}^{-2}$ do exist. Such high column densities have been detected only in high $z$ GRBs so far (GRB050904 at $z=6.29$ and and GRB060510B at $z=4.9$ ). These column densities imply a huge extinction of the rest-frame UV light, if dust with properties similar to those in the Galaxy, the SMC, or even for a dust with a grain distribution strongly shifted toward large grain sizes (Stratta et al. 2004, 2005) would be associated to the X-ray absorbing gas. The simple detection of the bright optical and near infrared afterglow of this GRB (Tagliaferri et al. 2005; Haislip et al. 2006; Boer et al. 2006) implies peculiar dust properties (Campana et al. 2006b; Stratta et al. 2007). Here we limit ourselves to note that high- $z$ GRBs with a gas column density similar to the one of GRB050904, but with less extreme dust properties, would easily remain undetected in the optical and near infrared. Furthermore, their host galaxies would be so faint that unambiguous associations with the GRB would be impossible, because the probability of finding such faint galaxies in the arcsec Swift XRT error-boxes would not be negligible, thus making it impossible to determine their redshift.

To assess more quantitatively how the different selection effects (peak flux limit, GRB obscuration, and magnitude of the optical afterglow) can modify the redshift distribution, we extracted from the Swift GRB sample a subsample having the same peak flux, $N_{\mathrm{H}}$ and Rmag (at $10 \mathrm{ks}$ ) distributions of the joined BeppoSAX+HETE2 sample (the "constrained" GRB sample hereafter). Figure 7 compares the redshift distribution of the constrained GRB sample with that of the full Swift and BeppoSAX+HETE2 GRB samples. To evaluate the uncertainty 
on the constrained GRB sample redshift distribution, we ran the random extraction 100 times and plotted the contours of the region covered by the constrained GRB sample redshift distributions. We see that the constrained GRB sample redshift distribution is consistent, to within the uncertainties, with the real BeppoSAX+HETE2 redshift distribution.

Other, more subtle, selection effects may be at work as well. For example, there are redshift ranges for which the typical interval covered by optical spectrometers $(\approx 3800-8000 \AA)$ does not contain any strong emission or absorption line. For example, strong emission lines such as $\mathrm{H} \alpha, \mathrm{H} \beta,[\mathrm{OIII}] \lambda \lambda 4959,5007$, [OII] $\lambda 3725$, are shifted outside the above wavelength range at $z \gtrsim 1$. , while Lyman- $\alpha$ enters the range at $z \sim 2.1$. The redshift range 1.1-2.1 is the so-called "redshift desert". Analogously, the strongest absorption feature after Lyman- $\alpha$ is the MgII $\lambda \lambda 2796,2803$ doublet. This is shifted in a region strongly affected by telluric features already at $z \gtrsim 1.5$, so redshift determinations through absorption lines in low signal to noise spectra are difficult in the redshift range 1.52.1. Treating these effects quantitatively (e.g. Bloom 2003) is complicated by the very diverse quality of the optical spectra of GRB afterglows. Unfortunately, because of the highly variable nature of these events, afterglow observations have often been performed in non-optimal conditions and instrument set-ups, and most importantly, they cannot be repeated.

\section{Conclusions}

We have compared three well-defined samples of long GRBs observed and localized by Swift (122 GRBs), BeppoSAX (39 GRBs) and HETE2 (44 GRBs), for a total of 205 objects. Secure spectroscopic redshifts were measured for 67 of these GRBs. The fraction of redshift determinations is similar in the three samples, $34 \%, 30 \%$, and $32 \%$, respectively.

Swift GRBs are, on average, slightly fainter and harder than BeppoSAX and HETE2 GRBs. This is probably due to both the higher sensitivity of the BAT detector with respect to the BeppoSAX and HETE2 detectors and to the higher energy range (15-150 keV), where Swift GRBs are detected and localized, compared to BeppoSAX and HETE2 $(\approx 2-20 \mathrm{keV})$. The distribution of the observer frame $N_{\mathrm{H}}$ for the Swift GRBs is shifted toward higher $N_{\mathrm{H}}$ values than BeppoSAX, at a confidence level of better than $98 \%$. This is again probably due to the different energy bands in which GRBs are localized by the two satellites. The most obscured GRBs have probably been missed by the BeppSAX survey. The distribution of the observer frame $N_{\mathrm{H}}$ for the Swift GRBs without redshift determination is also shifted toward higher $N_{\mathrm{H}}$ values than that of the Swift GRBs with a redshift determination (confidence level of better than 99\%), implying that the sample of Swift GRBs with redshift determinations is biased against high obscuration. This is confirmed by a more detailed analysis of the sample of bright GRBs. If dust is associated to the X-ray absorbing gas, one would expect that extinction makes the discovery and study of optical afterglows of bright Swift GRBs more difficult. This is probably the case, since the fraction of bright Swift GRB with redshift is only $29 \%$, while that of bright BeppoSAX and HETE2 GRBs is $62 \%$ and $50 \%$, respectively. Highly obscured, bright, low-redshift GRBs are likely to be present in the Swift sample, but so far most of them must have escaped redshift determination (we expect that the majority of the 17 bright Swift GRBs without redshift are at $z \lessgtr 2$ ). A program to discover and measure the magnitude and the redshift of the host galaxies of bright

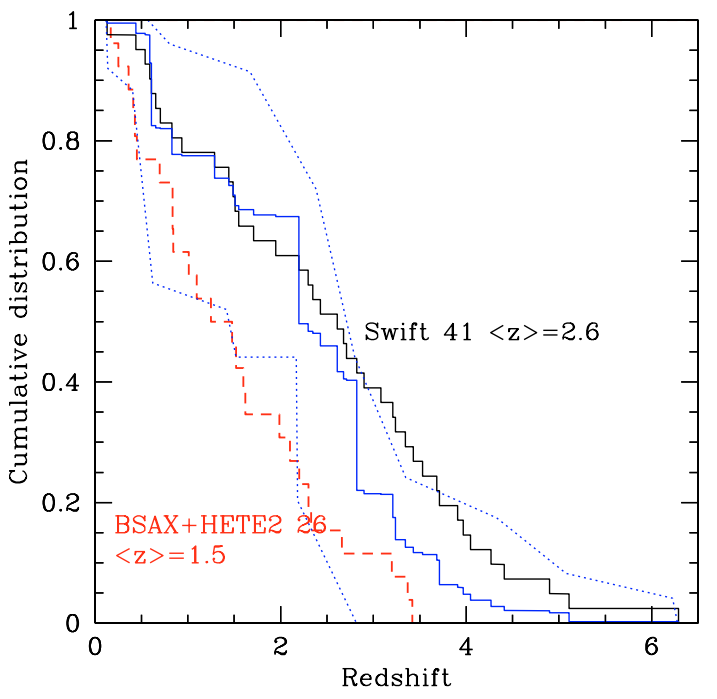

Fig. 7. The average cumulative redshift distribution of a subsample of Swift GRBs having the same peak flux, $N_{\mathrm{H}}$ and $R$ mag (at $10 \mathrm{ks}$ ) distributions of the joined BeppoSAX+HETE2 samples (thin solid line) compared with the Swift (thick solid line) and BeppoSAX+HETE2 (dashed line) total redshift distributions. The thin dotted lines mark the redshift range covered by 100 random extractions and give an idea of the statistical uncertainty associated to a single extraction of a redshift distribution of $26 \mathrm{GRBs}$ from a parent population.

Swift GRBs could confirm this conclusion and provide a sample of GRB redshifts unbiased against obscuration.

Highly X-ray obscured GRBs also do exist at high redshifts. The detection of bright optical and near-infrared (UV rest-frame) afterglows from these GRBs implies a dust-to-gas ratio and/or dust composition different from those of nearby GRBs (Stratta et al. 2007). Indeed, at $z \gtrsim 5$ the major source of dust in the local Universe (AGB stars) falls short of enough time to produce enough dust, implying that high- $z$ GRB host galaxies probably contains much less dust than lower redshift host galaxies. This implies that redshift determination of high- $z$ GRBs would not be more difficult than that of lower redshift GRBs, even if the observed optical and near-infrared bands sample the UV rest frame. If this is the case, the Swift sample of GRBs with redshifts would be a fair sample of the real high- $z$ GRB population.

The absence of high-redshift GRBs in the BeppoSAX and HETE2 samples of GRBs with measured redshift is most likely due to the fact that the median delay between the GRB event and the optical and near infrared follow-ups for BeppoSAX and HETE2 GRBs is $\sim 50$ times and $\sim 15$ times longer than for Swift GRBs. At the time of the BeppoSAX and HETE2 follow-up, the faint afterglow of high-redshift GRBs was too faint to allow redshift determination through absorption-line spectroscopy. Furthermore, the host galaxies of high-redshift GRBs are too faint to allow redshift determination through their emission lines. High-redshift GRBs may well be present in the BeppoSAX and HETE2 samples, but it is extremely difficult, if not impossible, to determine their redshift and therefore recognize them as such.

Swift optical afterglows, measured at a fixed observer-frame time, e.g. $10 \mathrm{ks}$ after the GRB event, are fainter than BeppoSAX and HETE2 optical afterglows, also when compared to the GRB 15-150 keV peak flux. This is somewhat surprising, because the higher median redshift of Swift GRBs implies that a fixed observer-frame time samples, on average, a shorter restframe time delay from the GRB event for the Swift GRBs than 
do BeppoSAX and HETE2. Because afterglows decrease like power laws, one would expect that the ratio between the GRB peak flux and the optical afterglow magnitude at a fixed observed time would be smaller for the Swift afterglows, contrary to what is observed. At least two effects may contribute to explaining the observed trend. The first is that at $z>4$ the Lyman- $\alpha$ forest enters the $R$ band, thus reducing the observed optical flux. The second is a higher extinction in Swift GRBs with respect to BeppoSAX and HETE2 GRBs, as disccused above.

To conclude, at least the selection effects on GRB localization and GRB redshift determination must be properly taken into account in order to safely use GRBs as cosmological tools and to derive the physical and cosmological evolution of the GRB formation rate from statistical analysis of the present GRB samples. This would allow a fair and quantitively-meaningful comparison with the star-formation rate estimated through other means. Moreover, star formation in regions hardly reachable by other techniques (low mass, dwarf galaxies, high-redshift galaxies, dust-enshshouded star formation sites) could be probed.

Acknowledgements. We thank Rosalba Perna and Elena Rossi for early discussions of the topics presented in this papers. We also thank Eli Waxman for useful comments and Luigi Stella for a careful reading of the manuscript. We acknowledge support from contracts ASI/I/R/039/04 and ASI/I/R/023/05/0.

\section{References}

Band, D. L. 2003, ApJ, 588, 945

Band, D. L. 2006, ApJ, 644, 378

Bloom, J. S. 2003, AJ, 125, 2865

Boer, M., Atteia, J. L., Damerdji, Y., Koltz, A., \& Stratta, G. 2006, ApJ, 638, L71
Campana, S., Romano, P., Covino, S., et al. 2006, A\&A, 449, 61

Campana, S., Lazzati, D., Ripamonti, E., et al. 2006, ApJL, in press [arXiv:astro-ph/0611305]

Daigne, F., Rossi, E. M., \& Mochkovitch, R. 2006, MNRAS, 372, 1034

D’Elia, V., Piranomonte, S., Ward, P., Fiore, F., Meurs, E. J. A., \& Norci, L. 2006, Proceedings of the meeting The Multicoloured Landscape of Compact Objects and their Explosive Origins, AIP, ed. L. Burderi, et al., in press

D'Elia, V., Fiore, F., Piranomonte, S., et al. 2007, A\&A, 467, 629

De Pasquale, M., Piro, L., Gendre, B., et al. 2006, A\&A, 455, 813

Dickey, J. M., \& Lockman, F. J. 1990, ARA\&A, 28, 215

Fynbo, J. P. U., Hjorth, J., Malesani, D., et al. 2007, Proceedings of the Eleventh Marcel Grossmann Meeting on General Relativity, ed. H. Kleinert, R. T. Jantzen, \& R. Ruffini [arXiv: astro-ph/0703458]

Guetta, D., Piran, T., \& Waxman, E. 2005, ApJ, 619, 412

Guetta, D., \& Piran, T. 2007, A\&A, submitted [arXiv: astro-ph/0701194]

Guetta, D., \& Della Valle, M. 2007, ApJL, in press [arXiv:astro-ph/0612194]

Jakobsson, P., Levan, A., Fynbo, J. P. U., et al. 2006, A\&A, 447, 897

Haislip, J. B., Nysewander, M. C., Reichart, D. E., et al. 2006, Nature, 440 181

Lockman, F. J., \& Condon, J. J. 2005, AJ, 129, 1968

MacFadyen, A. I., \& Woosley, S. E. 1999, ApJ, 524, 262

Natarajan, P., Albanna, B., Hjorth, J., et al. 2005, MNRAS, 364, L8

Piro, L., De Pasquale, M., Soffitta, P., et al. 2005, ApJ, 623, 314

Porciani, C., \& Madau, P. 2001, ApJ, 548, 522

Prochaska, J. X., Chen, H., \& Bloom, J. 2006, ApJ, 648, L97

Sakamoto, T., Lamb, D. Q., Kawai, N., et al. 2005, ApJ, 629, 311

Savaglio, S. 2006, New J. Phys., 8, 295 [arXiv: astro-ph/0609489]

Stratta, G., Fiore, F., Antonelli, L. A., Piro, L., \& De Pasquale, M. 2004, ApJ, 608,846

Stratta, G., Perna, R., Lazzati, D., et al. 2005, A\&A, 441, 83

Stratta, G., Maiolino, R., Fiore, F., \& D'Elia, V. 2007, ApJL, in press [arXiv:astro-ph/0703349]

Tagliaferri, G., Antonelli, L. A., Chincarini, G., et al. 2005, A\&A, 443, L1

Wijers, R. A. M. J., et al. 1998, MNRAS, 294, L13

Zeh, A., Klose, S., \& Kann, D. A. 2006, ApJ, 637, 889 